\title{
A Narrative Review on the Current Application of Biomarkers in the Management of Colorectal Cancer
}

\author{
Laurence Devoto', Deborah S Keller ${ }^{1,2}$ and Manish Chand ${ }^{1 *}$
}

${ }^{1}$ Department of Surgery, University College London Hospital, NHS Trust, London, UK

${ }^{2}$ Department of Surgery, Baylor University Medical Centre, Dallas, TX, USA

\begin{abstract}
Currently a wide range of biomarkers and potential biomarkers exist across a variety of fields for the management of colorectal cancer. These can be a specific molecule or a radiographical finding and can predict outcome or response to treatment. The field is being developed along several fronts with many new innovations happening in the last few years however few have made it into routine clinical practice with others requiring validation.

The evolving markers of significance are; MicroRNA, epithelial-to-mesenchymal-transition, imaging and metabolic with several sub-divisions depending on the pathway effected. This review will provide a narrative appraisal of our current understanding and clinical application of these biomarkers.
\end{abstract}

Keywords: Imaging biomarkers; Molecular biomarkers; Colorectal cancer diagnosis; Prognosis

\section{Background}

Colorectal cancer is one of the most common types of cancer, and leading causes of cancer-related deaths in both genders worldwide [1]. In 2017, there will be an estimated 135,430 new cases of colorectal cancer diagnosed, with 39,220 new cases of rectal cancer specifically [2]. Disease management for patients with colorectal cancer have improved significantly in the past 2 decades, and treatment plans have become more personalized to optimize care [3]. Biomarkers are a way to use more personalized information to improve patient care, help determine the patient's prognosis and guide the ideal treatment plan to improve cancer specific survival. The National Cancer Institute defines a biomarker as a biological molecule found in blood, other body fluids, or tissues that is a sign of a normal or abnormal process [4]. (NCI dictionary of cancer terms. Biomarker. Available online at: http:// www.cancer.gov/dictionary?cdrid=45618). In cancer, biomarkers can be objectively measured and evaluated as an indicator(s) of normal biological processes, or pharmacological responses to a therapeutic intervention [5,6]. The ideal biomarkers can help determininee predisposition for treatment, early detection of disease, and assessment of prognosis [7]. In this submission, we review the growing evidence for using biomarkers as a tool for these processes and responses in colorectal cancer. For this summation, the biomarkers are broadly grouped into metabolic, molecular, and imaging categories.

\section{Metabolic Biomarkers}

Colorectal cancer results from complex interactions between inherited susceptibility, inflammatory conditions, and environmental/ lifestyle-related risk factors, where diet and body habitus have a major impact. The hypothesis that diet and related metabolic, anthropometric and hormonal markers impacted cancer development was originally proposed in the 1940s [8]. Many studies that followed supported the association of elevated body mass index (BMI), metabolic disordersincluding hyperglycemia, hyperinsulinemia, dyslipidemia, type 2 diabetes, and hypertension and visceral adiposity with the risk of CRC and colon carcinogenesis [9]. Adipose tissue, which is viewed like glandular tissue, performing endocrine, paracrine and autocrine functions, has been found key in the process of neoplastic transformation, especially in inhibiting the anti-lipolytic effect of insulin [10-15]. Two specific hormonal systems, adipokines and the insulin/insulin-like growth factor (IGF) axis, are the most studied metabolic biomarkers of CRC $[16,17]$. The systems regulate processes including glucose and lipid metabolism, inflammation, angiogenesis, and cell proliferation [18]. Studies have found they markers in these systems impact risk clinical behavior, and mortality. To date, serum adiponectin, leptin, resistin and visfatin levels (and its receptors) are validated metabolic biomarkers of CRC. High concentrations of serum HDL are associated with a decreased risk of CRC, while increased circulating IGF1 levels and an increased IGF1/IGFBP3 ratio are associated with a higher risk of CRC. Hyperinsulinemia and hyperglycemia are associated with higher mortality from CRC, and increased HbAlC levels are an independent predictor of aggressive clinical behavior in CRC patients. Conversely, high IGF2 levels are associated with improved overall survival in CRC patients [17]. Further studies will determine the independent role of these possible biomarkers and their impact on rectal cancer specifically.

A hybrid of the molecular and metabolic biomarker fields is metabonomics. Metabonomics provides a systematic, time-dependent measurement of metabolic shifts occurring in response to drugs, environmental stimuli or disease [19-29]. It offers a functional view of system activity by showing all micromolecular data downstream of the genome and proteome [30]. This wider view of system activity past macromolecules such as DNA and RNA could facilitate personalized approaches for colorectal cancer diagnosis, prognosis and therapeutic personalisation $[22,28]$.

\section{Molecular Biomarkers}

Molecular biomarkers grew out of the pathways for colorectal cancer development. There are 3 distinct paths: chromosomal instability causing mutations in oncogenes, epigenetic methylation of varying genes, and

*Corresponding author: Manish Chand, Department of Surgery, University College London Hospitals, NHS Trust, 250 Euston Rd, Bloomsbury, London NW1 2BU, UK Tel: 020 34475879; Fax: 02034479218; E-mail: manish.chand@uclh.nhs.uk

Received June 09, 2017; Accepted June 24, 2017; Published June 26, 2017

Citation: Devoto L, Keller DS, Chand M (2017) A Narrative Review on the Current Application of Biomarkers in the Management of Colorectal Cancer. J Mol Biomark Diagn 8: 355. doi: 10.4172/2155-9929.1000355

Copyright: () 2017 Devoto L, et al. This is an open-access article distributed under the terms of the Creative Commons Attribution License, which permits unrestricted use, distribution, and reproduction in any medium, provided the original author and source are credited. 
microsatellite instability resulting from defective DNA repair. The epidermal growth factor receptor (EGFR) pathway has proved the most clinically relevant molecule involved in the chromosomal instability pathway. In fact, the development of biomarkers in colorectal cancer stemmed from the benefits demonstrated with KRAS and microsatellite instability testing $[10,11,23,24]$. Mutation of some of the components of KRAS on the EGFR pathway, including KRAS and NRAS codons 12 and 13 of exon 2, 59 and 61 of exon 3, and 117 and 146 of exon 4, render the malignant cells resistant to anti-EGFR therapies like Cetuximab and Panitumumab. This has a direct impact on patient care [25-27]. As a result, screening of KRAS/NRAS mutation status is mandatory prior to the start of treatment. This is also a therapeutic target, with monoclonal antibody therapies targeting the epidermal growth factor receptor (EGFR) that binds the EGFR extracellular domain, blocking EGFR signalling pathways; knowledge of the mutational status of genes in this pathway serve as predictive biomarkers of response to these therapies [25-27]. In the future, this could be an avenue for imaging biomarkers, as up regulation of EGF could potentially be measured. Other mutations in genes of the EGFR signalling pathways may affect response of CRC to anti-EGFR antibody therapies, including involving other exons of KRAS, NRAS, BRAF, PIK3CA, and PTEN. Further research will evolve on their role and guidelines addressing the molecular testing of EGFR pathway genes beyond KRAS.

Microsatellite instability (MSI) is a hypermutable phenotype caused by the loss of DNA mismatch repair activity. MSI is detected in about $15 \%$ of all colorectal cancers; $3 \%$ are of these are associated with Lynch syndrome and the other $12 \%$ are caused by sporadic, acquired hypermethylation of the promoter of the MLH1, MSH2, MSH6, or PMS2, most commonly [28]. MSI status also has a correlation with outcome, and MSI high or low is a useful molecular marker to stratify stage II colorectal cancer, as node negative colorectal cancer patients with MSI-high tumours have been shown to have a better outcome than patients with MSH-low tumours; adjuvant chemotherapy is usually not indicated in these cases $[29,30]$. Thus, it is recommended that clinicians should order mismatch repair status testing in patients with colorectal cancers for the identification of patients at high risk for Lynch syndrome and/or prognostic stratification [31].

MicroRNAs (miRNAs) are short, noncoding RNAs that regulate gene expression through post-transcriptional interactions with mRNA [32]. They have the potential to impact a vast range of downstream effects, and may act as either oncogenes or tumour suppressors, depending on the target proteins affected. Thus, miRNAs are directly involved in multiple biological pathways that can influence carcinogenesis and progression [33-35]. Their role has been described in breast and lung cancer, and continued work has shown mutated miRNAs have been found in multiple targets linked to cancerous transformation in general, such as p53 and EGFR [36-39]. Further exploration of the role of miRNA involvement has promise with as a miRNAs as a clinically relevant biomarker to predict tumour staging and response to treatment in colorectal cancer $[37,38]$.

\section{Imaging Biomarkers}

Imaging biomarkers provide a way to objectively measure tumour response to a therapeutic intervention and potentially detect early disease, in a non-invasive manner. The simplest imaging biomarker is tumour size, which can be reliably measured on CT or MRI $[40,41]$. Reduction in tumour size correlates with a positive response to neoadjuvant treatment and improved survival [42]. Using the blood flow on dynamic contrast enhanced CT or MRI scans as a surrogate for angiogenesis has been correlated with the development of metastases and decreased survival, and may have benefit as an imaging biomarker [43-46]. Other variables seen specifically on MRI following chemoradiotherapy, such as the degree of fibrosis, correlates with the histopathalogical tumour regression grade, and can be applied to determine the timing or need for surgical management [47]. Another variable on MRI, extramural venous invasion (EMVI), has been shown to be an independent indicator of prognosis and may be a valuable biomarker [48]

Specific CT and MRI types may hold additional benefit as a marker for response and prognosis. While T2 weighted MRI is the standard for evaluating staging and response to therapy in rectal cancer, the diffusion weighted imaging (DWI) sequences may add additional value for evaluation after response to chemoradiotherapy $[49,50]$. DWI analyses water molecules during MRI acquisition and can highlight cell death and vascular alterations typically before size changes occur [51-53] In addition, contrast enhanced CT measuring tumour heterogeneity could emerge as a biomarker for prognosis. Study has found tumours demonstrating less heterogeneity were associated with poorer survival, supporting the use texture analysis to staging contrast-enhanced CT, and possibly serve as a prognostic biomarker in colorectal cancer [54].

One method of measuring response is through metabolism, as with positron emission tomography (PET) scans. The concept of altered cancer metabolism, described as the Warburg effect, where cancer cells preferentially convert glucose into lactate even in the presence of abundant oxygen, has been validated in a variety of cancer subtypes [55-58]. This serves as the support for using [18F]-fluorodeoxyglucose (FDG) enhanced positron emission tomography (FDG-PET) imaging of solid tumours, which shows glycolytic flux [54,59]. Other modalities are harnessing this metabolic effect with other imaging tools to provide value, such as the combined PET/CT to predict early recurrence in the treatment of liver metastases, and application of PET/MR to provide information on tumour staging, response to neoadjuvant therapy, and disease recurrence $[60,61]$. Further studies are needed to define the true benefit of these studies in colorectal cancer care. The metabolism can be seen in a dynamic fashion, combining tissue specific spectra with chemometric data as a real-time imaging biomarker, as with high resolution nuclear magnetic resonance spectroscopy (HR-MAS NMR). To date, HR MAS NMR has shown reductions in lipids and glucose with increase of taurine, lactate and glycine in cancerous rectal mucosa [62]. This holds promise in developing targeted agents for rectal cancer [63].

\section{Conclusion}

Multiple types of biomarkers exist that have a variety of clinical applications, but few are currently used in routine practice. The ideal biomarkers for cancer have applications in determining predisposition, early detection, assessment of prognosis, and drug response [64]. However, much work is needed to fully develop these potentially useful markers. As the discovery, validation, and application of biomarkers continue to grow, future focused trials are required to determine their role in colorectal cancer management and outcomes.

\section{Conflict of Interests}

None

\section{Funding}

None

\section{References}

1. http://www.cancer.org/cancer/colonandrectumcancer/detailedguide/colorectalcancer-key-statistics 
2. Engstrom PF, Arnoletti JP, Benson AB, Chen YJ, Choti MA, et al. (2009) NCCN clinical practice guidelines in oncology: Rectal cancer. J Natl Compr Canc Netw 7: 838-881.

3. Schmoll HJ, Van Cutsem E, Stein A, Valentini V, Glimelius B, et al. (2012) ESMO consensus guidelines for management of patients with colon and rectal cancer: A personalized approach to clinical decision making. Ann Oncol 23 2479-2516.

4. Young PE, Womeldorph CM, Johnson EK, Maykel JA, Brucher B, et al. (2014) Early detection of colorectal cancer recurrence in patients undergoing surgery with curative intent: Current status and challenges. J Cancer 5: 262-271.

5. Strimbu K, Tavel JA (2010) What are biomarkers? Curr Opin HIV AIDS 5: 463-466

6. Biomarkers definitions working (2001) Biomarkers and surrogate endpoints: Preferred definitions and conceptual framework. Clin Pharmacol Ther 69: 89-95.

7. Tanaka T, Tanaka M, Tanaka T, Ishigamori R (2010) Biomarkers for colorectal cancer. Int J Mol Sci 11: 3209-3225.

8. Riboli E (2001) The European Prospective Investigation into Cancer and Nutrition (EPIC): Plans and progress. J Nutr 131: 170S-175S.

9. Aleksandrova K, Nimptsch K, Pischon T (2013) Influence of obesity and related metabolic alterations on colorectal cancer risk. Curr Nutr Rep 2: 1-9.

10. Ma Y, Yang Y, Wang F, Zhang P, Shi C, et al. (2013) Obesity and risk of colorectal cancer: A systematic review of prospective studies. PLoS One 8: e53916.

11. Prieto-Hontoria PL, Pérez-Matute $P$, Fernández-Galilea $M$, Bustos $M$, Martínez JA, et al. (2011) Role of obesity associated dysfunctional adipose tissue in cancer: A molecular nutrition approach. Biochim Biophys Acta 1807: 664-678.

12. Hursting SD, Dunlap SM (2012) Obesity, metabolic dysregulation, and cancer A growing concern and an inflammatory (and microenvironmental) issue. Ann N Y Acad Sci 1271: 82-87.

13. Greenberg AS, Obin MS (2006) Obesity and the role of adipose tissue in inflammation and metabolism. Am J Clin Nutr 83: 461S-465S.

14. Clayton PE, Banerjee I, Murray PG, Renehan AG (2011) Growth hormone, the insulin-like growth factor axis, insulin and cancer risk. Nat Rev Endocrinol 7: 11-24.

15. Naukkarinen J. (2008) Molecular background of common dyslipidemia Helsinki: National Public Health Institute. pp. 43-54

16. Giovannucci E (2007) Metabolic syndrome, hyperinsulinemia, and colon cancer: A review. Am J Clin Nutr 86: 30-34.

17. Muc-Wierzgoń M, Nowakowska-Zajdel E, Dzięgielewska-Gęsiak S, Kokot T, Klakla K, et al. (2014) Specific metabolic biomarkers as risk and prognostic factors in colorectal cancer. World J Gastroenterol 20: 9759-9774.

18. Trujillo ME, Scherer PE (2006) Adipose tissue-derived factors: impact on health and disease. Endocr Rev 27: 762-778.

19. Nicholson JK, Lindon JC, Holmes E (1999) Metabonomics: Understanding the metabolic responses of living systems to pathophysiological stimuli via multivariate statistical analysis of biological NMR spectroscopic data. Xenobiotica 29: 1181-1189.

20. Mirnezami R, Kinross JM, Vorkas PA, Goldin R, Holmes E, et al. (2012) Implementation of molecular phenotyping approaches in the personalized surgical patient journey. Ann Surg 255: 881-889.

21. Nicholson JK, Holmes E, Kinross JM, Darzi AW, Takats Z, et al. (2012) Metabolic phenotyping in clinical and surgical environments. Nature 491: 384-392.

22. Chen W, Zu Y, Huang Q, Chen F, Wang G, et al. (2011) Study on metabonomic characteristics of human lung cancer using high resolution magic-angle spinning $1 \mathrm{H}$ NMR spectroscopy and multivariate data analysis. Magn Reson Med 66: 1531-1540.

23. Phipps Al, Buchanan DD, Makar KW, Win AK, Baron JA, et al. (2013) KRAS mutation status in relation to colorectal cancer survival: The joint impact of correlated tumour markers. Br J Cancer 108: 1757-1764.

24. Rosty C, Young JP, Walsh MD, Clendenning M, Walters RJ, et al. (2012) Colorectal carcinomas with KRAS mutation are associated with distinctive morphological and molecular features. Mod Pathol 26: 825-834.

25. De Roock W, Claes B, Bernasconi D, De Schutter J, Biesmans B, et al. (2010) Effects of KRAS, BRAF, NRAS, and PIK3CA mutations on the efficacy of cetuximab plus chemotherapy in chemotherapy-refractory metastatic colorectal cancer: A retrospective consortium analysis. Lancet Oncol 11: 753-762.
26. De Roock W, De Vriendt V, Normanno N, Ciardiello F, Tejpar S (2011) KRAS, BRAF, PIK3CA, and PTEN mutations: Implications for targeted therapies in metastatic colorectal cancer. Lancet Oncol 12: 594-603.

27. Grothey A (2010) EGFR antibodies in colorectal cancer: Where do they belong? $\mathrm{J}$ Clin Oncol 28: 4668-4670.

28. Boland CR, Goel A (2010) Microsatellite instability in colorectal cancer. Gastroenterology 138: 2073-2087.

29. Funkhouser WK, Lubin IM, Monzon FA, Zehnbauer BA, Evans JP, et al. (2012) Relevance, pathogenesis, and testing algorithm for mismatch repair-defective colorectal carcinomas: A report of the Association for Molecular Pathology. J Mol Diagn 14: 91-103.

30. Kawakami H, Zaanan A, Sinicrope FA (2015) Implications of mismatch repairdeficient status on management of early stage colorectal cancer. J Gastrointest Oncol 6: 676-684.

31. Sepulveda AR, Hamilton SR, Allegra CJ, Grody W, Cushman-Vokoun AM, et al. (2017) Molecular biomarkers for the evaluation of colorectal cancer Guideline summary from the American Society for Clinical Pathology, College of American Pathologists, Association for molecular pathology, and American Society of Clinical Oncology. J Oncol Pract 13: 333-337.

32. Bartel DP (2004) MicroRNAs: Genomics, biogenesis, mechanism, and function Cell 116: 281-289.

33. Huang Q, Gumireddy K, Schrier M, le Sage C, Nagel R, et al. (2008) The microRNAs miR-373 and miR-520c promote tumour invasion and metastasis. Nat Cell Biol 10: 202-210.

34. Lee DY, Deng Z, Wang CH, Yang BB (2007) MicroRNA-378 promotes cel survival, tumor growth, and angiogenesis by targeting SuFu and Fus-1 expression. Proc Natl Acad Sci USA 104: 20350-20355.

35. Ma L, Teruya-Feldstein J, Weinberg RA (2007) Tumour invasion and metastasis initiated by microRNA-10b in breast cancer. Nature 449 : $682-688$.

36. Yu SL, Chen HY, Chang GC, Chen CY, Chen HW, et al. (2008) MicroRNA signature predicts survival and relapse in lung cancer. Cancer Cell 13: 48-57.

37. 37. Slaby O, Svoboda M, Michalek J, Vyzula R (2009) MicroRNAs in colorecta cancer: Translation of molecular biology into clinical application. Mol Cancer 8: 102.

38. Slaby O, Svoboda M, Fabian P, Smerdova T, Knoflickova D, et al. (2007) Altered expression of miR-21, miR-31, miR-143 and miR-145 is related to clinicopathologic features of colorectal cancer. Oncology 72: 397-402.

39. Schetter AJ, Leung SY, Sohn JJ, Zanetti KA, Bowman ED, et al. (2008) MicroRNA expression profiles associated with prognosis and therapeutic outcome in colon adenocarcinoma. JAMA 299: 425-436.

40. Benjamin RS, Choi H, Macapinlac HA, Burgess MA, Patel SR, et al. (2007) We should desist using RECIST, at least in GIST. J Clin Oncol 25: 1760-1764.

41. O'Connor JP, Jackson A, Asselin MC, Buckley DL, Parker GJ, et al. (2008) Quantitative imaging biomarkers in the clinical development of targeted therapeutics: Current and future perspectives. Lancet Oncol 9: 766-776.

42. Patel UB, Brown G, Machado I, Santos-Cores J, Pericay C, et al. (2017) MR assessment and outcomes in patients receiving neoadjuvant chemotherapy only for primary rectal cancer: Long-term results from the GEMCAD 0801 trial. Ann Oncol 28: 344-353.

43. Goh V, Glynne-Jones R (2014) Perfusion CT imaging of colorectal cancer. Br J Radiol 87: 20130811

44. George ML, Dzik-Jurasz AS, Padhani AR, Brown G, Tait DM, et al. (2001) Non-invasive methods of assessing angiogenesis and their value in predicting response to treatment in colorectal cancer. Br J Surg 88: 1628-1636.

45. Goh V, Halligan S, Wellsted DM, Bartram Cl (2009) Can perfusion CT assessment of primary colorectal adenocarcinoma blood flow at staging predict for subsequent metastatic disease? A pilot study. Eur Radiol 19: 79-89.

46. Hayano K, Shuto K, Koda K, Yanagawa N, Okazumi S, et al. (2009) Quantitative measurement of blood flow using perfusion CT for assessing clinicopathologic features and prognosis in patients with rectal cancer. Dis Colon Rectum 52: 1624-1629.

47. Mandard AM, Dalibard F, Mandard JC, Marnay J, Henry-Amar M, et al. (1994) Pathologic assessment of tumor regression after preoperative chemoradiotherapy of esophageal carcinoma. Clinicopathologic correlations. Cancer 73: 2680-2686 
Citation: Devoto L, Keller DS, Chand M (2017) A Narrative Review on the Current Application of Biomarkers in the Management of Colorectal Cancer. J Mol Biomark Diagn 8: 355. doi: 10.4172/2155-9929.1000355

48. Patel UB, Blomqvist LK, Taylor F, George C, Guthrie A, et al. (2012) MRI after treatment of locally advanced rectal cancer: How to report tumor response-the MERCURY experience. AJR Am J Roentgenol 199: W486-W495.

49. Sun YS, Zhang XP, Tang L, Ji JF, Gu J, et al. (2010) Locally advanced recta carcinoma treated with preoperative chemotherapy and radiation therapy: Preliminary analysis of diffusion-weighted MR imaging for early detection of tumor histopathologic downstaging. Radiology 254: 170-178.

50. Hein PA, Kremser C, Judmaier W, Griebel J, Pfeiffer KP, et al. (2003) Diffusionweighted magnetic resonance imaging for monitoring diffusion changes in rectal carcinoma during combined, preoperative chemoradiation: Preliminary results of a prospective study. Eur J Radiol 45: 214-222.

51. Koh DM, Brown G, Riddell AM, Scurr E, Collins DJ, et al. (2008) Detection of colorectal hepatic metastases using MnDPDP MR imaging and diffusionweighted imaging (DWI) alone and in combination. Eur Radiol 18: 903-910.

52. Taouli B, Chouli M, Martin AJ, Qayyum A, Coakley FV, et al. (2008) Chronic hepatitis: Role of diffusion-weighted imaging and diffusion tensor imaging for the diagnosis of liver fibrosis and inflammation. J Magn Reson Imaging 28: 89-95.

53. Ichikawa T, Erturk SM, Motosugi U, Sou H, lino H, et al. (2006) High-Bvalue diffusion-weighted MRI in colorectal cancer. AJR Am J Roentgeno 187: $181-184$

54. Ng F, Ganeshan B, Kozarski R, Miles KA, Goh V (2013) Assessment of primary colorectal cancer heterogeneity by using whole-tumor texture analysis: contrast-enhanced CT texture as a biomarker of 5-year survival. Radiology 266: 177-184.

55. Sitter B, Bathen TF, Singstad TE, Fjosne HE, Lundgren S, et al. (2010) Quantification of metabolites in breast cancer patients with different clinical prognosis using HR MAS MR spectroscopy. NMR Biomed 23: 424-431.
56. Kobayashi T, Nishiumi S, Ikeda A, Yoshie T, Sakai A, et al. (2013) A novel serum metabolomics-based diagnostic approach to pancreatic cancer. Cancer Epidemiol Biomarkers Prev 22: 571-579.

57. Zhang T, Wu X, Ke C, Yin M, Li Z, et al. (2013) Identification of potential biomarkers for ovarian cancer by urinary metabolomic profiling. J Proteome Res 12: 505-512.

58. Warburg O (1956) On the origin of cancer cells. Science 123: 309-314.

59. Reivich M, Alavi A (1993) Positron emission tomographic studies of loca cerebral glucose metabolism in humans in physiological and pathophysiological conditions. Adv Metab Disord 10: 135-176.

60. Cornelis F, Storchios V, Violari E, Sofocleous C, Schoder H, et al. (2016) $18 \mathrm{~F}-\mathrm{FDG} \mathrm{PET} / \mathrm{CT}$ is an immediate imaging biomarker of treatment success after liver metastasis ablation. J Nucl Med 57: 1052-1057.

61. Wiesmüller M, Quick HH, Navalpakkam B, Lell MM, Uder M, et al. (2013) Gall comparison of lesion detection and quantitation of tracer uptake between PET from a simultaneously acquiring whole-body PET/MR hybrid scanner and PET from PET/CT. Eur J Nucl Med Mol Imaging 40: 12-21.

62. Mirnezami R, Jimenez B, Li J, Veselkov K, Kinross J, et al. (2014) Rapid diagnosis and staging of colorectal cancer via high resolution magic angle spinning nuclear magnetic resonance (HR-MAS NMR) spectroscopy of intact tissue biopsies. Ann Surg 259: 1138-1149.

63. Jimenez B, Mirnezami R, Kinross JM, Cloarec O, Keun HC, et al. (2013) $1 \mathrm{H}$ HR-MAS NMR spectroscopy of tumour induced local metabolic "field-effects" enables colorectal cancer staging and prognostication. J Proteome Res 12 959-968.

64. Tanaka T, Tanaka M, Tanaka T, Ishigamori R (2010) Biomarkers for colorectal cancer. Int J Mol Sci 11: 3209-3225 